\title{
Influência da Wiireabilitação sobre o desempenho funcional no teste de caminhada de 6 minutos em idosos sedentários: um estudo piloto
}

\author{
Influence of Wiireabilitation on functional \\ performance in the 6-minute walk test in sedentary \\ elderly: a pilot study
}

\author{
Felipe Lima Rebêlo' \\ Fernanda Souza Soutinho ${ }^{2}$ (1) \\ Felipe César dos Santos Soares ${ }^{3}$ (1)
}

\section{Vitória Anjos da Silva ${ }^{4}$ (c) Anna Beatriz Santos Florêncio ${ }^{5}$ (1) Nicole Fernanda dos Santos Lima ${ }^{6}$ (1)}

${ }^{1}$ Autor para correspondência. Centro Universitário CESMAC (Alagoas). Maceió, Brasil. feliperebelo_fisio@yahoo.com.br, ${ }^{2-3}$ Centro Universitário CESMAC (Alagoas). Maceió, Brasil. fernandassoutinho08@gmail.com, felipecesar_s.soares@hotmail.com ${ }^{4-6}$ Universidade Estadual de Ciências da Saúde de Alagoas (Alagoas). Maceió, Brasil. vitoriaanjoss7@gmail.com, abeatrizsf23@gmail.com, nicole_limafe@hotmail.com

\begin{abstract}
RESUMO | INTRODUÇÃO: A incorporação de videogames como ferramentas auxiliares na prática da reabilitação possibilitou uma nova perspectiva de intervenção em diversas áreas, um deles é a Wiireabilitação, que tem se mostrado ferramenta eficaz em diversas perspectivas clínicas, sendo também utilizada na prática de atividade física, e, por sua característica lúdica, pode ser um importante aliado na prática de exercício físico em populaç̃̃es com baixa adesão, em especial, os idosos. OBJETIVO: Avaliar a influência da Wiireabilitação sobre a melhora do desempenho funcional no Teste de caminhada de seis minutos (TC6M) em idosos sedentários. MÉTODOS: Trata-se de um estudo piloto, onde foram incluídos idosos com 60 anos ou mais, de ambos os sexos e sedentários. Todos os idosos foram inicialmente avaliados pelo Teste de Caminhada de 6 minutos (TC6M). Posteriormente, procedeu-se com a intervenção aeróbica através da Wiireabilitação, tendo protocolo definido pelos pesquisadores. Foram programados três meses de intervenção, com frequência de três vezes por semana, e duração de 30 minutos por sessão. O TC6M foi realizado na metade do protocolo de intervenção e ao final dos três meses. Para análise e interpretação dos dados procedeu-se com a estatística descritiva. RESULTADOS: A amostra do estudo foi composta por 5 participantes, com predominância do sexo feminino $(N=4)$ e média de idade de 66,2 anos $\pm 6,27$. Na avaliação do TC6M todos os participantes obtiveram mais de 50 metros de ganho em relação ao pré-teste, evidenciando aumento no desempenho funcional. CONCLUSÃo: Para os pacientes aqui avaliados, a Wiireabilitação mostrou-se um recurso efetivo sobre o desempenho funcional no Teste de caminhada de 6 minutos.
\end{abstract}

PALAVRAS-CHAVE: Idosos. Inatividade Física. Condicionamento Aeróbico. Desempenho Funcional.
ABSTRACT | INTRODUCTION: The incorporation of video games as additional tools in the practice of rehabilitation has enabled a new perspective of intervention in several areas; one is Wiireahabilitation, which has proven to be an effective tool in several clinical perspectives, it is also used in the practice of physical activity, and because of its playful characteristic, can be an important ally in the practice of physical exercise in populations with low adherence, especially the elderly. OBJECTIVE: To evaluate the influence of Wiireahabilitation on improving functional performance in the six-minute walk test (6MWT) in sedentary elderly. METHODS: This is a pilot study, which included elderly people aged 60 or over of both sexes and sedentary. All the elderly were initially evaluated by the 6 -minute walk test (6MWT). Subsequently, an aerobic intervention was carried out through Wiireahabilitation, with a protocol defined by the researchers. Three months of intervention were programmed, with a frequency of three times a week and a duration of 30 minutes per session. The 6MWT was performed in the middle of the intervention protocol and at the end of the three months. For data analysis and interpretation, descriptive statistics were used. RESULTS: The study sample consisted of 5 participants, with a predominance of females $(N=4)$ and a mean age of 66.2 years \pm 6.27 . In the assessment of the $6 \mathrm{MWT}$, all participants obtained more than 50 meters of gain in relation to the pre-test, showing an increase in functional performance. CONCLUSION: For the patients evaluated here, Wiireahabilitation proved to be an effective resource on functional performance in the 6-minute walk test.

KEYWORDS: Elderly. Physical Inactivity. Aerobic conditioning. Functional Performance. 


\section{Introdução}

O processo de envelhecimento é determinado por uma multiplicidade de fatores que afetam a capacidade funcional, causando o declínio das funções orgânicas (morfológicas, psicológicas, bioquímicas e neuromusculares). Esse processo é influenciado pela variação de diversos fatores, tais como estilo de vida, condições socioeconômicas, aspectos genéticos, doenças crônicas entre outros. ${ }^{1,2}$

Esse declínio fisiológico leva à perda da capacidade funcional do idoso, afetando sua mobilidade, e o impede ou diminui a sua capacidade de realizar suas Atividades Básicas de Vida diária (ABVD) e Atividades Instrumentais de Vida Diária (AIVD). No entanto, pode ser mutável, a partir de uma assistência adequada à saúde, direcionando-o a um bom funcionamento físico, mental e social., 2,3

Diante disso, percebe-se a importância das práticas preventivas e intervencionistas que objetivem a promoção de um envelhecimento saudável, que prime pela manutenção da capacidade funcional, possibilitando ao idoso a realização de suas atividades com autonomia e independência. Nesse contexto, vários procedimentos de intervenção, seja ele preventivo ou reabilitador, estão disponíveis para que se alcancem tais objetivos, com destaque atual aos recursos da biotecnologia. 4,5

A utilização da tecnologia vem se tornando cada vez mais eficaz em diversos setores da área de saúde, tanto no âmbito preventivo quanto em reabilitação de doenças já instaladas, sendo incorporada também na realização de atividade física para fins terapêuticos ou recreativos, destacando-se as tecnologias de realidade virtual. ${ }^{6}$ Dentre essas tecnologias, vem tomando grande destaque a utilização dos exergames; entre eles, o Nintendo ${ }^{\circledR}$ Wii, um vídeo game que utiliza sensores que captam os movimentos realizados pelo usuário. Este recurso vem sendo utilizado como uma alternativa da prática de atividade física aeróbica de forma recreativa permitindo que os pacientes possam reduzir o sedentarismo e melhorar seu desempenho funcional de forma prazerosa e que irá trazer melhores resultados tanto físicos, quanto psicológicos devido ao entretenimento proporcionado, com o uso da ferramenta. Este é um recurso tecnológico utilizado até mesmo em unidades de terapia intensiva. ${ }^{7-9}$
Alguns pesquisadores concordam que essa prática é uma alternativa motivacional, principalmente para os que possuem dificuldades em aderir ou manter-se em programas de atividade física, tornando o exercício mais espontâneo e consequentemente a atividade mais prazerosa e agradável, de extrema relevância quando se fala na população idosa. ${ }^{10,11}$

Atualmente, alguns pesquisadores da área focam na temática da relação entre o Nintendo ${ }^{\circledR}$ Wii e a prática do exercício físico. As pesquisas mais recentes vêm mostrando que este instrumento é um grande aliado no combate e tratamento da obesidade, diminuindo riscos de morbidades, bem como na área da geriatria, onde demonstra respostas significativas, pois além de favorecer o condicionamento físico, melhora o equilíbrio, reduzindo o risco de quedas. ., 12

Embora as investigações científicas sobre a utilização deste game venham aumentando, ainda são limitados os estudos experimentais sobre esse tema, principalmente no que se diz respeito à efetividade desse recurso em programas de condicionamento aeróbico. $\frac{13,14}{14}$ Dentro dessa perspectiva, o presente estudo teve como objetivo avaliar a influência da Wiireabilitação sobre o desempenho funcional sobre o Teste de caminhada de seis minutos em idosos fisicamente inativos.

\section{Métodos}

Este artigo trata-se de um estudo piloto, aprovado pelo Comitê de ética em Pesquisa do Centro Universitário CESMAC, sob número de protocolo 1449/2012. Os dados foram coletados no período de agosto a dezembro de 2014. Antes do início da coleta, foi procedida a leitura e posterior assinatura do Termo de Consentimento Livre e Esclarecido para os voluntários da pesquisa. O estudo foi realizado no ambulatório de Fisioterapia motora da SUPRIMIDO. Os sujeitos foram recrutados no Grupo de Envelhecimento Ativo da Santa Casa (GESAC). O GEASC é um projeto de promoção e educação em saúde da Santa Casa de Misericórdia de Maceió. Acontece semanalmente, com duração de um ano, onde os idosos participantes recebem instruções da equipe multidisciplinar em forma de palestras e atividades dinâmicas direcionadas ao envelhecimento ativo. 
Os pesquisadores dirigiram-se ao espaço físico onde acontecem as reuniões do GEASC e foram explicados aos idosos quais seriam os objetivos da pesquisa e realizado o convite formal para participação da mesma. Foram adotados como critérios de inclusão indivíduos com idade igual ou superior a 60 anos de ambos os sexos, sem distinção de raça e cor. Foram excluídos indivíduos com distúrbios cardiovasculares, praticantes de atividade física regular; com distúrbio cognitivo; deficientes visuais e auditivos; idosos com distúrbios clínicos descompensados; aqueles com restrição médica para prática de atividade aeróbica e distúrbios osteoarticulares ou neurológicos que impossibilitem a postura ortostática e a interação com o recurso utilizado para a pesquisa (Nintendo® Wii).

Respeitando o limite temporal, limitações do espaço físico e a quantidade de equipamentos necessários para a coleta de dados, estabeleceu-se um número máximo de cinco participantes, que foram selecionados através de sorteio. Depois de selecionados, os indivíduos foram convocados a comparecerem no espaço físico onde funciona o ambulatório de fisioterapia motora da unidade hospitalar envolvida na pesquisa. No primeiro momento os idosos passaram por uma avaliação inicial, onde foram coletados dados socioeconômicos e demográficos e questões referentes à saúde. Após a coleta desses dados, os idosos foram submetidos ao Teste de caminhada de 6 minutos. Para a realização deste teste, os participantes foram orientados previamente a trajar roupas leves e calçado adequado. Os mesmos receberam todas as instruções para a realização do teste, que foi executado na presença constante dos pesquisadores, seguindo-se o protocolo recomendado para execução do mesmo. ${ }^{13}$

Todo o teste foi realizado num corredor de 30 metros. Os idosos foram instruídos previamente com o objetivo de obter-se a máxima compreensão da escala de percepção de esforço que foi realizada no $1^{\circ}, 3^{\circ}$ e $6^{\circ}$ minuto do teste. Antes de iniciar e após o término da avaliação foram monitorizados a frequência respiratória ( $F R)$, frequência cardíaca ( $\mathrm{FC}$ ), saturação periférica de oxigênio (SpO2) e pressão arterial (PA). A SpO2 foi verificada através do oxímetro de pulso portátil (Conect TM), durante a realização do teste. A PA mensurada através do esfigmomanômetro adulto com braçadeira de velcro (22-32cm, OMROMTM) e a FR foram aferidas antes e ao final do teste. No transcorrer dos 6 minutos foram anotados os números de voltas percorridas e ao final de cada teste, anotava-se o número de voltas dadas, assim como o número de metros da última volta, a fim de calcular a distância percorrida pelo voluntário. ${ }^{13}$

Após a avaliação inicial, foram agendados os dias de atendimento de cada idoso, sempre intercalados, com frequência de três vezes por semana, em dias alternados: segunda, quarta e sexta. O recurso utilizado para o treino aeróbico foi o videogame Nintendo ${ }^{\circledR}$ Wii, um console doméstico da Nintendo ${ }^{\circledR}$ que utiliza sensores para captar os movimentos realizados pelo usuário através do Wii Remote Plus (Figura 1), um controle, que integra um giroscópio mantido com o paciente durante o decorrer das atividades realizadas para a captação precisa de seus movimentos. Além disso, o mesmo utiliza uma câmera infravermelha, um pequeno alto falante para emissão de sons simples, e três acelerômetros embutidos. Outro dispositivo utilizado para captação de movimentos foi a Wii Balance Board (Figura 2), uma plataforma com sensores de pressão que captam os movimentos do usuário de acordo com as mudanças do centro de gravidade. $\underline{6}$

Figura 1. Demonstração das linhas vetoriais de captação de movimento do Wii Remote Plus

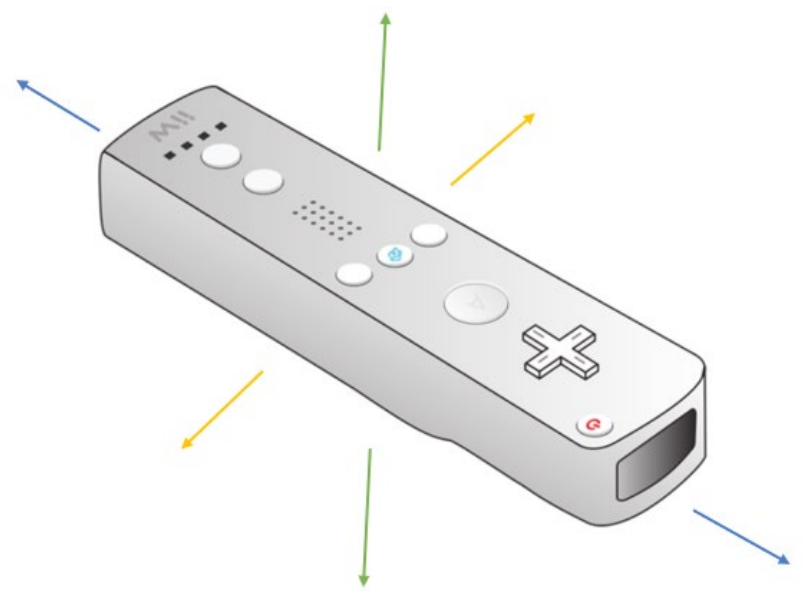

Fonte: https://pt.365psd.com/vector/wii-remote-clip-art-49907 


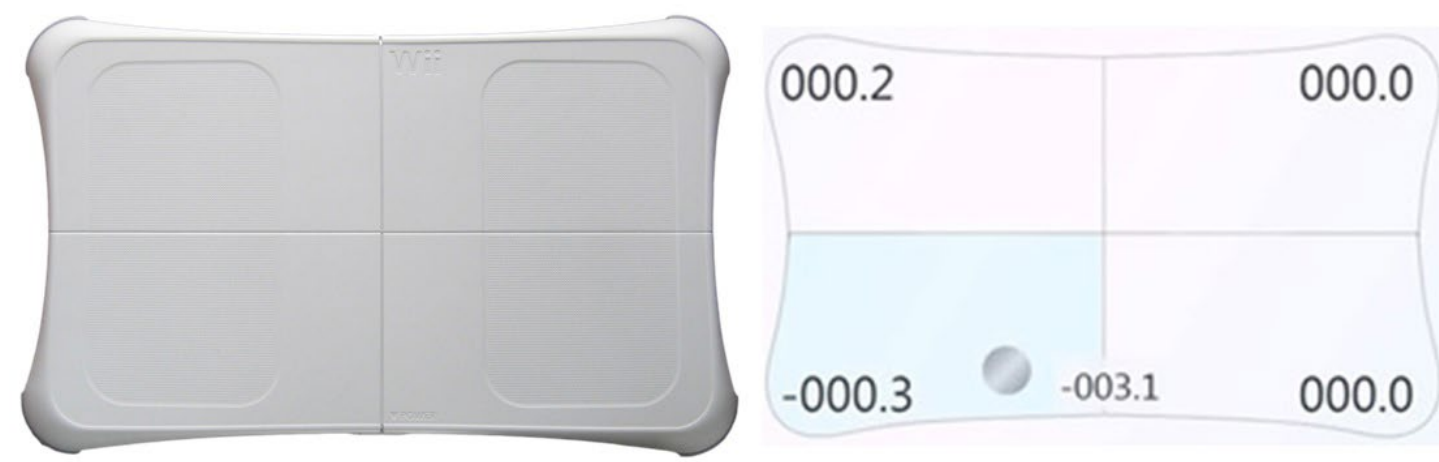

Fonte: Os autores (2021).

Os jogos utilizados faziam parte do pacote Wii Fit Plus, que possui um bloco de jogos direcionado para o treino aeróbico. O protocolo foi definido pelos pesquisadores, onde se programou três meses (12 semanas) de intervenção, com frequência de três vezes por semana, e duração de 30 minutos por sessão. O protocolo estabelecido respeitou o princípio do treino aeróbico, onde foram utilizados os jogos: Cabeceos, Footing, Boxing e Plataformas (Figura 3). A conduta era sempre iniciada com o jogo Cabeceos, objetivando o aquecimento e adaptação ao recurso. Este jogo simula a atividade esportiva do futebol, onde o indivíduo deve cabecear as bolas lançadas pelo oponente e/ou desviar dos possíveis obstáculos. É realizado com o paciente em ortostase sobre a plataforma de equilíbrio (Wii Balance Board). O mesmo realiza descarga de peso alternada em membros inferiores, em conjunto com inclinação latero-lateral de tronco quando interage com o game, esta etapa tinha duração de cinco minutos.

Figura 3. Representação gráfica dos jogos

\section{Cabeceos}

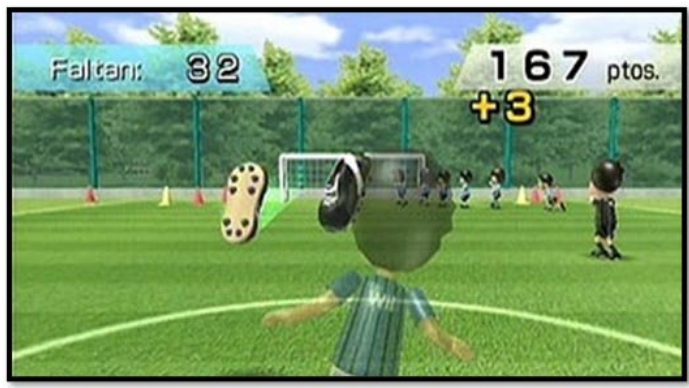

Boxing

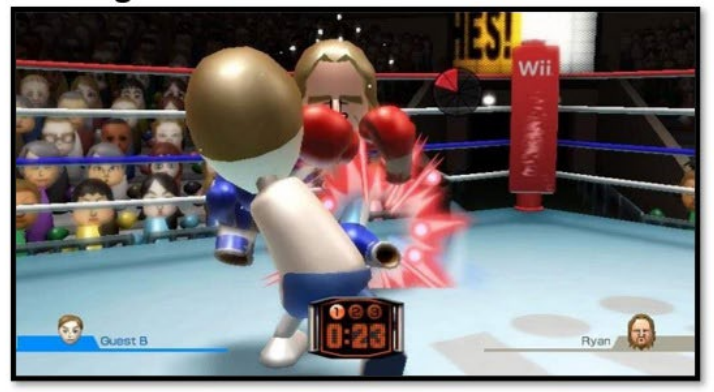

\section{Footing}

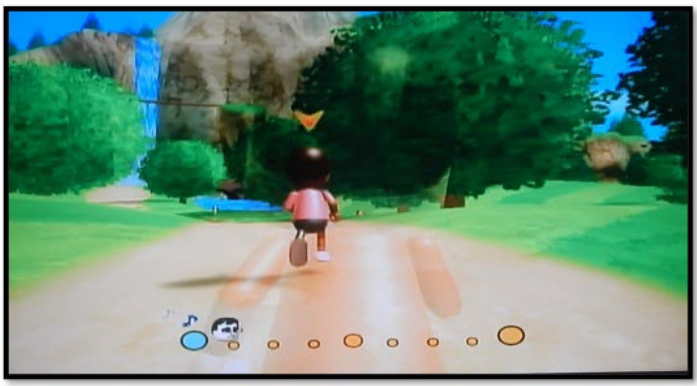

\section{Plataforma}

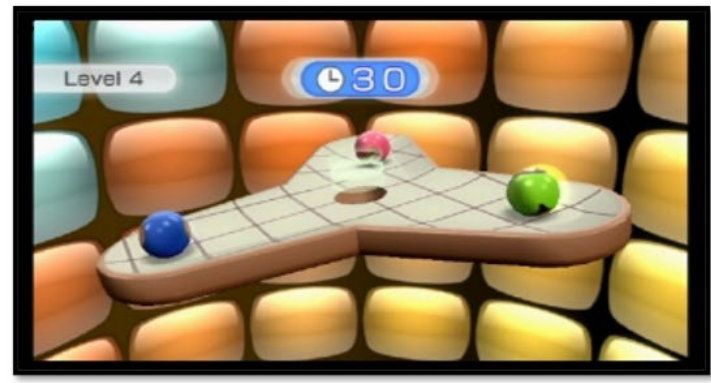

Fonte: Wii Fit Plus, Nintendo® Wii. 
Em seguida, procedia-se com os jogos aeróbicos, onde se optou pelos jogos Footing e Boxing, com duração de dez minutos cada, feito dentro da capacidade que o indivíduo alcançava. O Footing consiste em um jogo de corrida estática, onde o indivíduo em ortostase, com o controle (Wii Remote Plus) na altura do quadril, realiza flexão e extensão de quadril e joelhos simulando a atividade, sempre sendo estimulado pelo examinador para alcançar um melhor desempenho funcional. No Boxing o paciente se posiciona em ortostase, com base alargada, realizando flexão de ombro com flexão e extensão de cotovelos, segurando o controle (Wii Remote Plus), tentando nocautear o adversário que aparecer na tela do jogo no videogame.

Por fim, para o resfriamento era realizado o jogo Plataformas, com cinco minutos de exercício. Durante o jogo, paciente realiza descarga de peso em ortostase sob a plataforma de equilíbrio (Wii Balance Board), com intuito de encaçapar a bola. Todos os pacientes repetiram o TC6M na metade (ao final da $6^{a}$ semana) e ao final dos três meses (após a $12^{\mathrm{a}}$ semana) de intervenção, objetivando-se a comparação desses resultados com os da avaliação inicial. O protocolo do estudo está descrito na tabela 1. Para análise e interpretação dos dados procedeu-se com a estatística descritiva, estando os resultados dispostos em médias, proporções e frequências.

Tabela 1. Protocolo do estudo

\begin{tabular}{|c|c|}
\hline Etapa & Descrição \\
\hline Avaliação inicial & $\begin{array}{l}\text { Coleta dos dados socioeconômicos e demográficos e questões } \\
\text { referentes à saúde. }\end{array}$ \\
\hline $\begin{array}{l}\text { Avaliação da Capacidade funcional relacionada a resposta ao } \\
\text { exercício em esforço submáximo }\end{array}$ & Aplicação do Teste de caminhada de 6 minutos. \\
\hline Aquecimento e adaptação ao recurso & $\begin{array}{l}\text { Jogo Cabeceos ( } 5 \text { minutos). Simulação da atividade de cabecear } \\
\text { bolas, nas diversas direções, bem como, desviar de } \\
\text { obstáculos, alternando a descarga de peso em membros } \\
\text { inferiores e realizando inclinação lateral de tronco, para } \\
\text { ambos os lados. Jogo realizando em ortostase com a } \\
\text { supervisão do terapeuta. }\end{array}$ \\
\hline Primeiro Treinamento & $\begin{array}{l}\text { Jogo Footing (10 minutos). Com o controle Wii remote Plus no } \\
\text { bolso, o indivíduo realiza simulação de corrida estática, } \\
\text { acompanhando seu trajeto na projeção do jogo. O terapeuta } \\
\text { supervisiona o exercício, corrigindo possíveis compensações, } \\
\text { estimulando o indivíduo ao seu melhor desempenho, sempre } \\
\text { monitorando os sinais vitais e Escala de Borg, no início, no } \\
\text { quinto minuto e ao final do exercício. }\end{array}$ \\
\hline Segundo Treinamento & $\begin{array}{l}\text { Jogo Boxing ( } 10 \text { minutos). Com os controles nas maõs, o } \\
\text { indivíduo simula golbes do boxe, mantendo a flexão de } \\
\text { ombros e realizando a flexão e extensão de cotovelos, } \\
\text { tentando, constantemente, nocautear o adversário virtual. O } \\
\text { terapeuta supervisiona o exercício, corrigindo possíveis } \\
\text { compensações, estimulando o indivíduo ao seu melhor } \\
\text { desempenho, sempre monitorando os sinais vitais e Escala de } \\
\text { Borg, no início, no quinto minuto e ao final do exercício. }\end{array}$ \\
\hline Resfriamento & $\begin{array}{l}\text { Jogo Plataformas (5 minutos). Indivíduo em ortostase, } \\
\text { realizando descarga e transferência de peso, dentro do limite } \\
\text { de estabilidade, na tentativa de encaçapar bolas dentro do } \\
\text { orifício de uma plataforma virtual. Jogo realizando com a } \\
\text { supervisão do terapeuta. }\end{array}$ \\
\hline $\begin{array}{l}\text { Avaliação da Capacidade funcional relacionada a resposta ao } \\
\text { exercício em esforço submáximo }\end{array}$ & $\begin{array}{l}\text { (Re)avaliação do Teste de caminhada de } 6 \text { minutos após o final } \\
\text { da } 6^{\text {a }} \text { semana de intervenção. }\end{array}$ \\
\hline $\begin{array}{l}\text { Avaliação Final da Capacidade funcional relacionada a } \\
\text { resposta ao exercício em esforço submáximo }\end{array}$ & $\begin{array}{l}\text { (Re)avaliação do Teste de caminhada de } 6 \text { minutos após o final } \\
\text { da } 12^{\mathrm{a}} \text { semana de intervenção. }\end{array}$ \\
\hline
\end{tabular}

Fonte: Os autores (2021). 


\section{Resultados}

A amostra do estudo foi composta por 5 indivíduos, onde identificou-se predominância do sexo feminino ( $\mathrm{N}=4)$. A média de idade encontrada foi de 66,2 $\pm 6,27$ anos, sendo a mínima de 60 e máxima 74 anos. A maioria dos participantes avaliados apresentou alta escolaridade (80\%) e renda maior que 1 salário mínimo (100\%).

No que diz respeito ao estado de saúde, todos os participantes relataram conviver com menos de duas comorbidades, não praticavam atividade física, referiram em sua maioria, fazer uso de apenas uma medicação e perceberam a saúde como boa; Todos os dados referentes as características socioeconômicas e demográficas e de saúde estão descritos na tabela 2.

Tabela 2. Distribuição da frequência das variáveis socioeconômicas, demográficas e de saúde

\begin{tabular}{|c|c|c|c|}
\hline Variável & Categorias & Frequência & $\%$ \\
\hline \multirow{2}{*}{ Sexo } & $M$ & 1 & 20,0 \\
\hline & $\mathrm{F}$ & 4 & 80,0 \\
\hline \multirow[t]{2}{*}{ Faixa etária } & $<70$ anos & 3 & 60,0 \\
\hline & $\geq 70$ anos & 2 & 40,0 \\
\hline \multirow[t]{3}{*}{ Escolaridade } & $<4$ anos & 0 & 0 \\
\hline & $5-9$ anos & 1 & 20,0 \\
\hline & $>9$ anos & 4 & 80,0 \\
\hline \multirow[t]{2}{*}{ Renda } & 1 Salário & 0 & 0 \\
\hline & > 1 Salário & 5 & 100 \\
\hline \multirow[t]{2}{*}{ Número de doenças } & $<2$ doenças & 5 & 100 \\
\hline & $\geq 3$ doenças & 0 & 0 \\
\hline \multirow[t]{3}{*}{ Medicação } & Até 1 & 3 & 60,0 \\
\hline & 2 & 1 & 20,0 \\
\hline & $\geq 3$ & 1 & 20,0 \\
\hline \multirow[t]{2}{*}{ Atividade Física } & Não & 5 & 100 \\
\hline & Sim & 0 & 0 \\
\hline \multirow[t]{2}{*}{ Percepção de Saúde } & Ruim & 0 & 0 \\
\hline & Boa & 5 & 100 \\
\hline
\end{tabular}

Fonte: Os autores (2021).

Quanto à avaliação do Teste de caminhada de 6 minutos, foram encontrados resultados satisfatórios na relação pré e pós-teste, onde todos os participantes obtiveram mais de 50 metros de ganho em relação ao pré-teste. Os resultados podem ser observados na Tabela 3.

Tabela 3. Distribuição dos resultados da avaliação pré e pós do Teste de caminhada de 6 minutos em metros e percentual de ganho

\begin{tabular}{|c|c|c|c|c|c|}
\hline Variável & & Pré intervenção & Após $6^{a}$ semana & Após $12^{\mathrm{a}}$ semana & $\%$ \\
\hline \multirow{5}{*}{ TC6M } & Participante 1 & 471,4 & 494,30 & 521,4 & 110 \\
\hline & Participante 2 & 573,1 & 600,9 & 624,2 & 108 \\
\hline & Participante 3 & 460,0 & 510,2 & 594,0 & 129 \\
\hline & Participante 4 & 525,0 & 577,0 & 625,0 & 119 \\
\hline & Participante 5 & 503,7 & 555,4 & 621,6 & 121 \\
\hline
\end{tabular}

Fonte: Os autores (2021). 


\section{Discussão}

A pesquisa aqui apresentada, que se trata de um estudo piloto, avaliou o efeito de um treinamento de condicionamento físico de idosos através da Wiireabilitação sobre o Teste de caminhada de $6 \mathrm{mi}-$ nutos, que avalia a capacidade funcional relacionada a resposta ao exercício em esforço submáximo. Os resultados dos cinco idosos participantes da pesquisa mostraram um aumento na distância percorrida durante o teste, mostrando que foi possível alcançar resultados positivos no desfecho avaliado, nesses participantes. Todos os idosos avaliados obtiveram um aumento superior a cinquenta metros.

O recurso utilizado na implementação do treinamento para o confinamento físico foi o Nintendo ${ }^{\circledR}$ Wii, um dispositivo considerado de realidade virtual não imersiva que se destaca como recurso lúdico na implementação de diversas terapias, ${ }_{-1,14}^{6}$ sendo foco de estudo de pesquisadores da área da atividade física, que avaliam os efeitos da utilização desse recurso sobre os componentes de interação corporal, os aspectos motivacionais, e os estudos comparativos desta prática com as práticas convencionais não associadas à realidade virtual. .5

Nesse sentido, apesar do protocolo desse estudo ter tido direcionamento para o condicionamento físico, a melhora alcançada pelos cinco participantes desse estudo piloto pode ser atribuída a ganhos em diversas habilidades, como otimização da força muscular, mobilidade e equilíbrio corporal. Nesse sentido, destaca-se o estudo de Contreras et al. $\frac{16}{\text { que avaliaram }}$ a força muscular e o risco de queda em idosos sedentários através da utilização do Nintendo ${ }^{\circledR}$ Wii e verificaram que a prática realizada através desse recurso provoca no indivíduo melhoras em relação às mudanças fisiológicas advindas do processo de envelhecimento, que podem ser percebidas de diversas maneiras, a curto e a longo prazo.

Os autores verificaram que os participantes apresentaram melhora na força muscular, equilíbrio, controle postural e, consequentemente, diminuição no risco de queda. O recurso serviu ainda como estímulo para atividade física regular por ser também entretenimento, propiciando um melhor bem-estar e consequente impacto positivo na qualidade de vida.
Outro aspecto que deve ser levado em consideração são os jogos utilizados no protocolo de pesquisa, que envolviam simulação de corrida e luta, atividades que proporcionam importante gasto energético, uma vez que promovem a movimentação dos membros superiores e também à utilização dos membros inferiores, em maiores velocidades, nos quais grandes grupamentos musculares são exigidos. ${ }^{17}$

Nesse sentido, destaca-se o estudo de Guérios ${ }^{18}$, que em sua tese de doutorado, realizou objetivou fazer uma análise comparativa do consumo de oxigênio (VO2) e da resposta da frequência cardíaca entre uma corrida em esteira ergométrica e corrida estática utilizando o game Wii Fit. Após análise dos dados, verificou-se uma diferença significativa entre os níveis de VO2 nas duas atividades, com valores superiores para esteira ergométrica. Já a FC não demonstrou diferença estatística. No entanto, a autora conclui que as duas atividades podem ser consideradas vigorosas. $\frac{18}{}$

Não foram encontrados muitos estudos com o direcionamento aqui abordado na população idosa. No entanto, os exercícios e o protocolo estabelecido para o treino aeróbico foram realizados de acordo com os princípios do treinamento aeróbio. Assim, os ganhos esperados para um treino convencional em ambiente real, já amplamente elucidados pela literatura, poderiam ser atribuídos para o treinamento virtual, com a vantagem do aspecto motivacional possibilidade pelo feedback constante fornecido pelo Nintendo ${ }^{\circledR}$ Wii.

Nessa perspectiva, destaca-se que o treino aeróbio promove vários ajustes no sistema muscular e cardiovascular, como um aumento no número e tamanho das mitocôndrias. O aumento da rede capilar tem um papel importante na melhora da capacidade aeróbia facilita o transporte e extração muscular de oxigênio, contribuindo assim para um maior consumo de oxigênio máximo e desempenho físico. 19

Como já mencionado, outro fator que poderia justificar o ganho na distância percorrida no Teste de Caminhada dos participantes dessa pesquisa é que o programa de condicionamento implementado também foi capaz de melhorar a força muscular e a contratilidade, principalmente em membros inferiores, o que em idosos é importante, diante do processo fisiológico de perda de massa muscular, principalmente naqueles com alterações respiratórias. ${ }^{20}$ 
Além disso, o incremento de força ora relatado também poderia interferir na velocidade da marcha desses indivíduos, sendo esta, outra variável que tem influência importante sobre a distância percorrida. Logo o aumento da velocidade resulta no aumento da distância obtida, representando uma forte correlação do condicionamento cardiovascular e muscular periférico exibido pelos indivíduos. ${ }^{21}$

A incorporação de vídeo games como ferramentas auxiliares na prática de atividade física e reabilitação possibilitou uma nova perspectiva de intervenção nessas áreas, por se tratar de uma ferramenta terapêutica lúdica, motivacional, que aumenta a adesão à terapia, sendo o Nintendo ${ }^{\circledR}$ Wii um dos recursos mais utilizados.

Destaca-se, no entanto, que estudos de maior rigor científico são necessários para que a evidência acerca do efeito dessa terapia sobre o desfecho aqui avaliado seja firmada na literatura. Esse estudo apresenta limitações, principalmente por se tratar de um estudo piloto, com um reduzido número de participantes e a ausência de um grupo controle, o que pode ampliar o risco de viés. Portanto, apesar de resultados importantes que possivelmente podem sugerir efeitos positivos da utilização da Wiireabilitação sobre a melhora no desempenho no teste de caminhada, frente ao incremento funcional, é preciso que novos estudos, como ensaios clínicos randomizados, sejam realizados.

\section{Conclusão}

Para os pacientes aqui avaliados, a Wiireabilitação mostrou-se um recurso efetivo para o aumento no desempenho funcional do Teste de caminha de $6 \mathrm{mi}-$ nutos após o treino com a terapêutica de condicionamento, sendo capaz de influenciar positivamente no resultado no Teste de caminhada de 6 minutos.

\section{Contribuições dos autores}

Soutinho FS, Soares FCS, Silva VA, Florêncio ABS, Lima NFS participaram da concepção e análise dos dados e redação do artigo. Silva VA participou da redação do artigo científico.

\section{Conflitos de interesses}

Nenhum conflito financeiro, legal ou político envolvendo terceiros (governo, empresas e fundações privadas, etc.) foi declarado para nenhum aspecto do trabalho submetido (incluindo, mas não se limitando a subvenções e financiamentos, participação em conselho consultivo, desenho de estudo, preparação de manuscrito, análise estatística, etc.).

\section{Referências}

1. Silva FLC, Santana WR, Rodrigues TS. Envelhecimento ativo: o papel da fisioterapia na melhoria da qualidade de vida da pessoa idosa: revisão integrativa. Uningá journal [Internet]. 2019;56(S4):134-144. Disponível em: http://revista.uninga.br/ index.php/uninga/article/view/2321

2. Costa NP, Polaro SHI, Vahl EAC, Gonçalves LHT. Contação de história: tecnologia cuidativa na educação permanente para o envelhecimento ativo. Rev Bras Enferm. 2016;69(6):1132-9. https://doi.org/10.1590/0034-7167-2016-0390

3. Garcia PA, Dias JMD, Dias RC, Santos P, Zampa CC. Estudo da relação entre função muscular, mobilidade funcional e nível de atividade física em idosos comunitários. Rev. bras. Fisioter. 2011;15(1):15-22. https://doi.org/10.1590/S1413$\underline{35552011000100005}$

4. Silva Júnior, JLA. Prática de exergame em dupla x individual: efeitos na adesão ao exercício físico e na capacidade funcional de idosos [dissertação] [Internet]. Passo Fundo: Universidade de Passo Fundo; 2019. Disponível em: http://tede.upf.br/jspui/ handle/tede/1865

5. Silva S, Tenório JM, Pisa IT. Quarta geração das tecnologias de apoio ao idoso para ambientes de vida assistida. Re. Saúd. Digi. Tec. Edu. 2019;4(1):74-84. https://doi.org/10.36517/resdite. v4.n1.2019.a6

6. Sousa FH. Uma revisão bibliográfica sobre a utilização do Nintendo ${ }^{\circ}$ Wii como instrumento terapêutico e seus fatores de risco. Revista Espaço Acadêmico [Internet]. 2011;11(123):15560. Disponível em: https://periodicos.uem.br/ojs/index.php/ EspacoAcademico/article/view/13045

7. Crapanzani TD. Realidade Virtual Na Prevenção De Quedas em Idosos [Internet]. Tubarão: Universidade do Sul de Santa Catarina; 2019. Disponível em: https://www.riuni.unisul.br/ bitstream/handle/12345/8962/realidade\%20virtual\%20na\%20 preven\%c3\%a7\%c3\%a30\%20de\%20quedas\%20em\%20idosos. pdf? sequence $=2 \&$ isAllowed $=y$

8. Graham CA, Perron RM, Feldman JR, Hall EE. Does Exergaming Achieve the Same Levels of Fitness Intensity as Unstructured Activity? Undergraduate Research Journal for the Human Sciences. 2011;10(1). Disponível em: https://www.kon.org/urc/v10/graham. html 
9. Penko AL, Barkley JE. Motivation and Physiologic Responses of Playing a Physically Interactive Video Game Relative to a Sedentary Alternative in Children. Ann Behave Med. 2010;39(2):162-9. https://doi.org/10.1007/s12160-010-9164-x

10. Oliveira D, Ladeira Â, Giacomin L, Pivetta N, Antunes M, Batista $\mathrm{R}$, et al. Depressão, autoestima e motivação de idosos para a prática de exercícios físicos. Psicol saúde doenças [Internet]. 2019;20(3):803-12. Disponível em: https://www.researchgate.net/ profile/Jose-Roberto-Nascimento-Junior/publication/338725184 Depression_self-esteem_and_motivation_of the_elderly_to practice_physical_exercises/links/5e2b50474585150ee780915f/ Depression-self-esteem-and-motivation-of-the-elderly-to-practicephysical-exercises.pdf

11. Ferreira D, Gil H. Envelhecer mais saudável com Exergames?!... efeitos, vantagens e limitações na utilização de jogos da nintendo Wii ® com idosos institucionalizados. In: Pocinho R, Carrana P, Navarro-Pardo E, Pereira AF, Margarigo C, Santos R, et al. Envelhecimento como perspectiva futura. Pamplona: Editorial Aranzandi; 2019. p. 797-808

12. Straker LM, Campbell AC, Jensen LM, Metcalf DR, Smith AJ, Abbott RA, et al. Rationale, design and methods for a randomised and controlled trial of the impact of virtual reality games on motor competence, physical activity, and mental health in children with developmental coordination disorder. BMC Public Health. 2011;11:654. https://doi.org/10.1186/1471-2458-11-654

13. Fernandes PM, Pereira NH, Santos ACBC, Soares MESM. Teste de Caminhada de Seis Minutos: avaliação da capacidade funcional de indivíduos sedentários. Rev Bras Cardiol [Internet]. 2012;25(3):185-91. Disponível em: http://www.onlineijcs.org/ english/sumario/25/pdf/v25n3a04.pdf

14. Magna TS, Brandão AF, Fernandes PT. Intervenção por realidade virtual e exercício físico em idosos. J Health Inform [Internet]. 2020;12(3):77-82. Disponível em: http://www.jhi-sbis. saude.ws/ojs-jhi/index.php/jhi-sbis/article/view/727

15. Rabelo AG, Costa S, Cardoso A, Lamounier E, Andrade AO. Uso de realidade virtual no tratamento de sintomas da doença de Parkinson: uma revisão sistemática [Internet]. XI Simpósio de Engenharia Biomecânica; 2018; Uberlândia. Disponível em: https://www.even3.com.br/anais/xiseb/128311/
16. Contreras KA, Udagawa M, Nonino F. Aplicação do Nitendo Wii em idosos sedentários na melhora da força muscular e diminuição do risco de quedas [Internet]. IX Mostra Interna de Trabalhos de Iniciação Científica e II Mostra Interna de Trabalhos de Iniciação em Desenvolvimento Tecnológico e Inovação; 2018; Maringá. Disponível em: http://rdu.unicesumar.edu.br/ handle/123456789/2056

17. Silva AF. Resposta fisiológica durante a prática de exergames: uma revisão de literatura [monografia] [Internet]. Santa Catarina: Universidade do Sul de Santa Catarina; 2018. Disponível em: https://www.riuni.unisul.br/handle/12345/5276

18. Guérios L. Análise comparativa do consumo de oxigênio e da resposta da frequência cardíaca entre uma corrida em esteira ergométrica e uma corrida virtual, em adultos jovens do sexo masculino [tese] [Internet]. Curitiba (PR): Pontifícia Universidade Católica do Paraná; 2012. Disponível em: http://bdtd.ibict.br/ vufind/Record/P PR e4ee72112c83f3623f1ea4ed43786d43

19. Janning PR. Adaptação mitocondrial induzida pelo exercício físico aeróbico: desvendando novos mecanismos moleculares [tese] [Internet]. São Paulo: Escola de Educação Física e Esporte; 2017. Disponível em: https://teses.usp.br/teses/ disponiveis/39/39132/tde-03012018-094243/pt-br.php

20. Torres SH, Montes MO, Loeb E, Mata A, Hernández N. Gender and skeletal muscle characteristics in subjects with chronic obstructive pulmonary disease. Respir Med. 2011;105(1):88-94. https://doi.org/10.1016/j.rmed.2010.05.010

21. Reis JM, Costa GC, Silva CTM, Leon EB. Avaliação de força muscular periférica e desempenho de membros inferiores após protocolo fisioterapêutico em idosos institucionalizados: projeto piloto [Internet]. Manaus: Universidade Federal do Amazonas; 2016. Disponível em: http://riu.ufam.edu.br/bitstream/ prefix/5275/2/J\%C3\%A9ssica\%20Melo\%20dos\%20Reis.pdf 